\title{
PROPUESTA DE UN MODELO DE SISTEMATIZACIÓN DE LA PRÁCTICA EN EL ÁMBITO DE LA INTERVENCIÓN SOCIAL A PARTIR DE LA INVESTIGACIÓN ACCIÓN PARTICIPACIÓN (IAP)
}

\author{
$M^{a}$ Cinta Guinot ${ }^{1}$, Mabel Segú ${ }^{1} y$ Patricia Espinosa ${ }^{2}$ \\ ${ }^{1}$ Departamento de Trabajo Social y Sociología. San Sebastián. - Universidad de Deusto, España cguinot@deusto.es \\ msegu@deusto.es \\ ${ }^{2}$ Departamento de Psicología Social y del Desarrollo Bilbao. Universidad de Deusto, España \\ patricia.espinosa@deusto.es
}

\begin{abstract}
Resumen. En el ámbito de la investigación cualitativa, en los procesos de investigación, donde que se asientan en la metodología y en los fundamentos de la investigación acción participativa (IAP), los y las investigadoras son facilitadores/as del proceso de reflexión y construcción colectiva y actores que interpretan procesos y favorecen la co-construcción y sistematización del conocimiento que surge de la práctica. La Responsabilidad Social Universitaria (RSU) promueve la producción y el uso del conocimiento para responder a las exigencias de los cambios sociales, estableciendo relaciones con el tejido social más próximo y respondiendo a sus demandas. A partir de nuestra experiencia investigadora, presentamos una propuesta de modelo emergente, para trabajar con equipos de intervención psicosocial, que permite acompañar desde la sistematización y facilitación sus prácticas, apoyando el logro de sus objetivos, dotando de metodología al trabajo que realizan y construyendo procedimientos reflexionados para mejorar sus propias prácticas, todo ello acompañado de opciones teóricas que las sustentan.
\end{abstract}

Palabras clave: IAP; Facilitación; Sistematización; Modelo; Equipo Psicosocial

PROPOSAL FOR A PRACTICE SYSTEMATIZATION MODEL IN THE FIELD OF SOCIAL INTERVENTION FROM THE PARTICIPATION ACTION RESEARCH (PAR)

\begin{abstract}
In the field of qualitative research, researchers are facilitators in the process of reflection and collective construction, as well as actors that interpret processes and promote co-construction and systematization of knowledge arising from practice in the research processes based on the methodology and the fundamentals of participatory action research (PAR). University Social Responsibility (USR) promotes the production and use of knowledge to meet the demands of social change, establishing relationships with the closest social structure and responding to their demands. From our research experience, we present an emerging model proposal to work with psychosocial intervention teams, which helps them develop their practices based on a systematization and facilitation process, supporting the achievement of their objectives, providing a methodology to their work and building reflected procedures to improve their own practices, all accompanied by theoretical options that support them.
\end{abstract}

Keywords: IAP; Facilitation; Systematization; Model; Psychosocial Team 


\section{INTRODUCCIÓN}

\subsection{El contexto de la investigación cualitativa}

Nos parece importante contextualizar de dónde surge el modelo que a continuación presentamos. El equipo de investigación está formado por Trabajadoras Sociales y Psicólogas que, a partir de las metodologías cualitativas de investigación, acompañan en los procesos de co-construcción de conocimiento a los equipos psicosociales de diferentes entidades del tercer sector o asociaciones que requieran de una sistematización de su práctica y demanden nuestros servicios. La experiencia del equipo de investigación en este tipo de acompañamientos ha permitido la emergencia de un modelo de sistematización de la práctica que es la que a continuación presentamos, con la voluntad de poder ser transferido a otros equipos y en otros ámbitos pues los resultados obtenidos son muy satisfactorios para todos los agentes participantes.

Es importante incidir en el hecho de que esta investigación se inscribe en la Universidad de Deusto donde la Responsabilidad Social Universitaria, se va consolidado como un aspecto transversal que recorre su triple misión en la docencia-formación, investigación-transferencia y proyección social. En este sentido desde el departamento de Trabajo Social y Sociología de la Universidad de Deusto, se entiende que la Universidad, a través de la investigación aplicada, tiene que dar respuestas a las necesidades sociales que las entidades del sector demandan, con la responsabilidad de prestar un servicio hacia la ciudadanía, siendo conscientes de la capacidad transformadora que la producción científica generada puede tener. Como equipo investigador, nos caracterizamos por liderar proyectos participativos a partir de diseños de Investigación Acción Participación, favoreciendo espacios compartidos que propicien la coconstrucción entre los equipos psicosociales y el propio equipo investigador, con la finalidad de generar no sólo un conocimiento sino un valor compartido (Porter \& Kramer, 2011), a partir de la sistematización de las prácticas desarrolladas mediante la facilitación y la sistematización de experiencias.

\subsection{Investigación basada en los principios de la Responsabilidad Social Universitaria}

Como indicamos, el modelo psicosocial que presentamos es fruto de las distintas investigaciones desarrolladas por el equipo de investigación, Deusto Valores Sociales, reconocido como equipo A por el Gobierno Vasco (2019), y dentro del ámbito Universitario 
de la Universidad de Deusto, donde hay un compromiso por dar sentido ético y social a todas aquellas actividades que se produzcan o deriven de su seno.

La Responsabilidad Social, en general, hace referencia al compromiso que cualquier entidad, institución o empresa tiene hacia la sociedad, en aras de incidir positivamente en la promoción y mejora de esta. En la actualidad la responsabilidad social se considera un concepto normativo no obligatorio por lo que no es de obligado cumplimiento por parte de las empresas, sino que es una voluntad ética a la que las diferentes instituciones pueden adherirse para que el fin último de éstas no sólo se base en el lucro, sino que incida en el progreso social, promoviendo las condiciones sociales y medioambientales de la comunidad donde opera.

En las Universidades, tanto a nivel de gestión como en el ámbito de la docencia y la investigación, está emergiendo esta nueva visión empresarial con mirada ética y comprometida y específicamente desde la Universidad de Deusto, alineados con los Objetivos de Desarrollo Sostenible (ODS) se empieza a valorar y gestionar los impactos tanto psicosociales como políticos y medioambientales, que se pueden producir derivados de cualquier proceso universitario y concretamente la repercusión que pudieran tener las distintas investigaciones que puedan desarrollarse en su seno. De hecho, La Responsabilidad Social Universitaria (RSU) es uno de los proyectos estratégicos del Plan Deusto 2018: Servicio y Compromiso (Universidad de Deusto, 2020). De esta manera, la toma de decisiones sobre qué temáticas abordar en las investigaciones han de ser bien valoradas pues van a tener su repercusión tanto de manera directa como indirecta, bien sea por acción o por omisión. La responsabilidad social de la Universidad de Deusto, es concebida como un proceso a través del cual se logra que la comunidad universitaria se interese por los problemas sociales de su contexto social más próximo e integre académicamente su comprensión en la generación de conocimiento y que aspire a la transformación social, cultural y económica de las estructuras de su territorio en términos de justicia y equidad social (Domínguez Pachón, 2009)., incidiendo en el papel y relaciones de las universidades con el entorno. Como obra de la Compañía de Jesús, la Universidad de Deusto renueva y concreta así su misión de servicio a la sociedad, promoviendo una ciudadanía responsable y comprometida, consciente de los problemas sociales y de su capacidad de transformar la realidad. 


\subsection{La facilitación en los procesos de Investigación Acción Participación}

Aplicando los principios de la RSU, las investigaciones desarrolladas por el equipo Deusto Valores Sociales, tienen como finalidad, la mejora de las condiciones sociales trabajando para y con las personas y comunidades de su territorio, facilitando la co-construcción de conocimiento en procesos de intervención psicosocial, desde una perspectiva hermenéutica y crítica, que la investigación cualitativa permite realizar científicamente.

En la actualidad, en el campo de la investigación en las Ciencias Sociales, son cada vez más las investigaciones que se diseñan a partir de las metodologías cualitativas, cuando su propósito es la consecución de avances teóricos y cambios sociales simultáneamente (Lewin, 1946), propiciando un cambio social transformador de la realidad y donde las personas tomen conciencia de su papel en ese proceso de transformación (Sandín, 2003).

Tal y como señalamos, el enfoque de investigación cualitativa, se ubica en los marcos epistemológicos de lo interpretativo y crítico que parten de las premisas de que los participantes que están viviendo una situación/problema son los que más conocen su realidad para poder abordarla y proponer soluciones que propicien el cambio o las mejoras; Además, se entiende que las dinámicas que se generan, dentro del marco de la investigación están vinculadas a los elementos culturales de la propia entidad en su entorno territorial y que el/la investigador/a acompaña, facilita y guía en todo el proceso pero el protagonismo es de los participantes (Segú \& González, 2019). En la Investigación Acción Participativa (IAP), los y las investigadoras son entendidos/as como investigadores/as en acción, actores/as facilitadores/as que asumen dos roles fundamentales: en primer lugar, el de facilitador del proceso de reflexión y construcción colectiva y, en segundo lugar, como actor que interpreta el proceso y la correspondiente construcción y sistematización del conocimiento.

Así en la investigación acción participativa (IAP) el investigador pasa a ser un facilitador de procesos, participando tanto en la construcción del conocimiento compartido como en garantizar una dinámica óptima del proceso. Este tipo de investigación nos acerca a trabajar con las personas y no sólo para o por las personas y pone en valor prácticas colaborativas de reciprocidad que permiten construir y aprender de manera colectiva. (Ferran, Guinot, Berasategui, 2019). Esta facilitación fomenta que los y las actoras de un proceso de IAP puedan reflexionar, decidir y actuar. De sus acciones depende la resolución de los 
problemas y conflictos que pretenden abordar y generar estrategias para buscar alternativas a esos problemas mediante su participación en procesos de reflexión y acción. (Costamagna y Larrea ,2017). Un requisito para la facilitación consiste en crear y mantener un espacio de trabajo colaborativo y capaz de ser generador de confianza, en el que todas las personas participantes se sientan seguras, estén dispuestas a compartir sus visiones, experiencias, y abiertas a aprender juntas, construyendo un contexto en el que se respeten y asuman las diferencias (Amnistía Internacional, 2011).

En cada proyecto de Investigación Acción Participación, sus tres componentes se combinan en proporciones variables. León y Montero (2002) hablan de un proceso de investigación con pasos "en espiral" donde se investiga al mismo tiempo que se interviene.

a) La investigación consiste en un procedimiento reflexivo, sistemático, controlado y crítico que tiene por finalidad estudiar algún aspecto de la realidad, de la situación problema con una expresa finalidad práctica.

b) La acción no sólo es la finalidad última de la investigación, sino que ella misma representa una fuente de conocimiento, al tiempo que la propia realización del estudio es en sí una forma de intervención.

c) La participación supone que en el proceso están involucrados no sólo los/as investigadores/as, sino la comunidad destinataria del proyecto, que no son considerados como simples objetos de investigación sino como sujetos activos que contribuyen a conocer y transformar su propia realidad (Zabala, 2005-2006).

El equipo facilitador se encarga de generar condiciones para que se desarrollen con garantías los procesos participativos favoreciendo la reflexión, compartiendo con los participantes las reflexiones co-construidas, pero sin tomar decisiones que correspondan a las y los propios participantes. Así, al equipo facilitador le corresponde tomar las decisiones asociadas a la propia tarea facilitadora, pero no aquellas otras que pertenecen a las y los agentes participantes. Para Fucks (2009), el facilitador es considerado un "artesano de contextos" un profesional que acompaña y cuida el proceso, ayudando a generar dinámicas y condiciones que permitan la participación de los miembros del grupo en todo el proceso, poniendo a su disposición sus recursos personales y técnicos para fomentar la eficiencia en la toma de decisiones y el logro de sus objetivos. La misión de un facilitador es aportar sus competencias para la creación de organizaciones flexibles, creativas, y resilientes en las que 
las personas sean vistas como una riqueza y no como un problema, construyendo condiciones para la autoorganización grupal (Perlo, De la Riestra, Costa,2009).

De manera que la función de facilitación en los proyectos de investigación aplicada desde la perspectiva de la IAP se centra, fundamentalmente, en promover la corresponsabilidad, estimulando la implicación e interacción de los distintos actores que participan en el proceso para compartir experiencias y diseñar buenas prácticas en el tema concreto que les ocupa. En el rol de facilitación, la función de acompañamiento es central y requiere el manejo de técnicas de diálogo generativo, negociación, construcción de consenso y generación de acuerdos. (Ferran, Guinot, Berasategui, 2019).

\section{LA SISTEMATIZACIÓN DE LA SISTEMATIZACIÓN: EL MODELO}

En el ámbito de la investigación, la sistematización, supone poner en valor la recuperación de opiniones, saberes y percepciones de las personas que intervienen en procesos de transformación e intervención social, planteado por tanto una perspectiva diferente a la posición academicista más clásica que entiende como única fuente de generación de conocimiento la producción teórica. En este sentido, Caparrós, Carbonero y Raya (2017) citando a Martinic (1984), indican que la sistematización permite vincular la teoría con la práctica desde una perspectiva dialéctica y representa un proceso de reflexión, capaz de ordenar y/o organizar los resultados de un proyecto. Así la sistematización se entiende como un proceso de generación de conocimiento desde la práctica vivida, de la cual se pueden extraer aprendizajes que contribuyan a enriquecer y mejorar la propia práctica de intervención. Por lo que con la sistematización se pretende captar los elementos clave en el desarrollo de una práctica, recuperar saberes, opiniones, formas de hacer y conocimientos de las personas participantes, así como detectar elementos que permitan mejorar nuevas experiencias. En la disciplina del Trabajo Social, la sistematización se entiende como un proceso participativo que ordena la experiencia de la intervención social, permite interpretarla y a la vez aprender nuevos conocimientos que puedan ser difundidos y compartidos. Además, tal y como plantean Caparrós, Carbonero y Raya (2017) la sistematización ordena los aspectos clave del proceso y la reflexión realizada durante el mismo. Por último, una vez sistematizada la experiencia ésta se convierte en un instrumento para la reflexión de todos los agentes implicados. 
La sistematización se conceptualiza como una forma de generación de conocimientos adecuada a las condiciones de trabajo y capacidades particulares de las personas que involucradas en la ejecución de las acciones y que están situadas en la práctica y en la acción, por ello uno de los retos es el de definir metodologías de producción de conocimientos adecuadas a la forma particular de pensar que tienen los profesionales de la acción, (Caparrós, Carbonero y Raya 2017). La sistematización de experiencias, por tanto, contribuye a mejorar la práctica profesional y a la generación de propuestas teóricometodológicas. Ya que al analizar la experiencia se abren posibilidades para comprender las prácticas cotidianas que suceden dentro de una determinada estructura organizativa, permitiendo comprender las prácticas que allí acontecen (Zavalla,2010). De este modo, la sistematización de experiencias aporta una doble contribución: mejorar la práctica de los equipos y enriquecer las reflexiones y propuestas teórico-conceptuales. Se considera que, en primer lugar, aporta mejoras en la práctica ya que permite al equipo obtener una visión común sobre el proceso, sus límites y posibilidades, y de este modo se potencia más cohesión y coherencia grupal. En segundo lugar, a partir de los aprendizajes obtenidos, contribuye a que los equipos y profesionales puedan superar el activismo y evitar de este modo la repetición mecánica de procedimientos.

En el campo teórico y del conocimiento, la sistematización también propicia el debate y la discusión, así como el enriquecimiento y la actualización de los conceptos y enfoques teóricos en los que se sustenta la práctica. Este acercamiento entre teoría y práctica se realiza a través de la acumulación y contrastación de los aprendizajes que derivan de experiencias particulares, ayudando a construir un saber colectivo a partir del cual, es posible llegar a producir teoría y metodología, (Marfil y Morgan 1995). De manera procesual en la sistematización se establecen cuatro momentos o fases: Reconstruir y describir la experiencia seleccionada para sistematizar; Analizarla/interpretarla de manera crítica; Extraer aprendizajes y generar nuevo conocimiento; Difundir y compartir lo aprendido (Caparrós, Carbonero y Raya, 2017).

\subsection{Una propuesta metodológica para construir un modelo de sistematización de la práctica}

Basándonos en la sistematización y en la figura de la facilitación de procesos antes expuestas, y tomando la IAP como marco de referencia, desde diferentes experiencias de investigación realizadas, presentamos un modelo emergente, procesual y sistémico que 
permite acompañar a equipos y organizaciones en la sistematización de sus práctica , apoyando el logro de sus objetivos, dotando de metodología al trabajo que realizan y construyendo procedimientos reflexionados para mejorar sus propias prácticas, todo ello acompañado de opciones teóricas que lo sustentan.

En las siguientes líneas pasamos a describir una propuesta de sistematización de la práctica configurada por una serie de fases y una secuencia de actividades que se van dando en el proceso y que configuran un ciclo, señalando que este proceso nunca es lineal. Cabe subrayar que en el ciclo de la sistematización de la práctica siempre se da una interrelación de los distintos componentes que lo configuran, ya que en cada momento van surgiendo situaciones no previstas, nuevas reflexiones, aportaciones, teorizaciones ... que cambian el rumbo de los objetivos previstos y esto conlleva que no sea posible separar en fases totalmente estancas esta propuesta. Por lo tanto, en coherencia con la IAP, este no es un modelo o esquema metodológico cerrado, sino que exige plantear algunos criterios que nos hagan avanzar en la creación de contextos investigativos más abiertos y procesuales, de modo que los propios resultados de la investigación se reintroducen en el mismo proceso para profundizar (Jara,2012). A continuación, se expone la especificidad de cada una de las fases, con sus respectivas orientaciones metodológicas:

\subsection{1 - A. Fase Inicial de Contextualización o Preparatoria}

A partir de una de una demanda o necesidad sentida por una organización o un equipo, se establece un encuentro entre miembros del equipo investigador y miembros de la organización en el que se clarifican las expectativas y las demandas para co-construir y definir un objeto de trabajo. Es aquí importante prestar a atención a los puntos de vista de cada participante, así como concretar los intereses y objetivos. En todo proceso intervienen diversas personas o grupos, algunos con una participación más directa y otros con una influencia más indirecta. La sistematización debe procurar captar la opinión y los puntos de vista de la mayor cantidad de actores posibles (Tapella y Rodríguez, 2014). También es relevante en este primer encuentro, abarcar lo posible y realizable para no generar frustración ni falsas expectativas y garantizar una viabilidad del proyecto. De esta manera:

1. Elaboración de una propuesta inicial El equipo investigador elabora una propuesta inicial teniendo en cuenta la demanda planteada y se genera un nuevo encuentro con los participantes para realizar propuestas y aclaraciones a la propuesta inicial. En este momento del proceso se requiere acotar y 
dimensionar lo que es factible, teniendo en cuenta las variables tiempo y recursos disponibles, esto favorece el logro de los objetivos y permite dimensionarlos (Caparrós, Carbonero y Raya, 2017).

2. Consensuar la propuesta El equipo investigador incorpora las aportaciones y los cambios sugeridos en ese encuentro y a partir de esa reelaboración se define el proyecto de investigación, concretando el objeto, los objetivos y la metodología. Este diseño siempre estará abierto a posibles cambios que surjan en el proceso de trabajo. El documento propuesto es consensuado y co-construido por los demandantes y por el equipo investigador. Este es un paso metodológico importante ya que la sistematización debe procurar captar la opinión y los puntos de vista de la mayor cantidad de actores posibles, como forma de garantizar la participación y compromiso en el proceso (Zúñiga y Zúñiga ,2013).

\section{Definición y clarificación de: objetivos, metodología de trabajo, tiempos, expectativas,}

limitaciones. Si entendemos la sistematización como un proceso de construcción de conocimientos sobre la práctica es necesario entonces definir un objeto de conocimiento, es decir, determinar qué se va a sistematizar y, por tanto, cuál es el eje de la sistematización. Además, es necesario delimitar el problema o aspecto de la experiencia que se quiere trabajar, identificando y explicitando aquellas variables y preguntas más significativas para la sistematización. En esto consiste la identificación y delimitación del objeto de conocimiento.

\subsection{2 - A. Fase de Desarrollo de la Experiencia}

Esta fase del proceso se inicia con la configuración de un contexto de trabajo relacional entre el equipo investigador y el equipo de la organización. Con la finalidad de observar, visualizar y analizar la propia práctica. Se establece un calendario de trabajo y contenidos para cada encuentro acordado.

A partir de estructurar el contexto, el equipo investigador elabora un diseño del proceso de trabajo implementado las siguientes técnicas de investigación para su puesta en marcha. Visualizar, observar y analizar la propia práctica: Mediante el trabajo compartido y colaborativo, desde la facilitación, planteando preguntas, promoviendo la narración de experiencias y de puntos de vista, dando espacio para las diferentes visiones, reflexionando sobre la práctica y analizando fortalezas y debilidades (Perlo, de la Riestra y Costa,2009), distinguiendo sus componentes, (éticos, epistemológicos, metodológicos), identificando los puntos fuertes y las debilidades, estableciendo preguntas que abran posibilidades, clarificando las relaciones (entre el equipo, con otros agentes, grupos, organizaciones, instituciones), reconociendo su funcionalidad y su impacto (Jara, 2012).Para analizar la práctica y poder sistematizarla el equipo investigador se apoya las siguientes técnicas: 
Diseño de las entrevistas grupales; planteando temáticas o preguntas para centrar las reuniones y promoviendo la reflexión para dar voz a todos los actores participantes.

Grabación autorizada (firma documento confidencialidad) y transcripción literal de las entrevistas grupales; para garantizar que todos los relatos y visiones tengan cabida en la investigación.

Análisis de contenido de las entrevistas grupales; utilizando la herramienta ATLAS.ti, permitiendo de este modo el análisis de las dimensiones y variables significativas para ordenar el discurso y analizar la práctica.

Análisis documental y bibliográfico de los conceptos y temáticas abordadas, para vincular teoría y práctica, dotándola de este modo de rigor científico. La revisión de la documentación es una actividad central del proceso de sistematización. Consiste en teorizar desde la práctica dotando de rigor a la experiencia práctica.

Elaboración de documentos borradores; desde la perspectiva del trabajo colaborativo, estos son documentos abiertos para generar debate en el grupo de trabajo, dando lugar al Feedback, favoreciendo la implementación de aportaciones a los documentos y matices que los participantes consideran adecuados incluir.

Todo ello con la finalidad de que la práctica pueda ser ordenada, interiorizada y apropiada, puesto que el objetivo más relevante es mejorarla, transformarla y generar buenas prácticas para que puedan ser reproducidas (FAO, 2004). En toda esta fase la función de facilitación cobra gran importancia, ya que el equipo investigador tiene que generar condiciones que permitan reflexionar, decidir y actuar a las personas participantes del proceso. De modo que la facilitación favorece la resolución de conflictos y genera estrategias para buscar alternativas a los problemas planteados en los grupos mediante procesos de reflexión y acción (Costamagna y Larrea 2017).

\subsection{3 - A. Fase de Cierre y Transferencia}

Todo proceso de sistematización debe arrojar un producto. Desde el comienzo el producto de la sistematización debe ser comunicado y comunicable (Tapella y Rodríguez,2014). Una sistematización no termina con la descripción de la evolución y de los resultados del proyecto. La sistematización es el proceso para identificar, capturar y documentar aprendizajes y experiencias claves con el propósito de transferir y adaptar el conocimiento. De esta forma la sistematización facilita la reflexión y el aprendizaje institucional, al posibilitar que el conocimiento y el aprendizaje derivados de los proyectos contribuyan a las 
decisiones y cambios institucionales, al tiempo que fomentan organizaciones más abiertas y reflexivas (FAO,2004).

En esta fase se realiza un Análisis e interpretación crítica de la experiencia, se elaboran conclusiones y recomendaciones y se elaboran productos para compartir los aprendizajes, definiendo la mejor forma de comunicarlos. Para cerrar el ciclo que configura el modelo que estamos proponiendo se elabora un informe final, el mismo tiene que estar consensuado para luego ser socializado. Los resultados del proceso de sistematización tienen que ser socializados con las mismas personas que han participado en el proceso. Como ya se ha señalado se puede ir realizando una devolución del documento en algunos momentos del proceso para recoger y retroalimentar el trabajo. La creatividad a la hora de comunicar los hallazgos es tan importante como el proceso de sistematización en sí mismo.El trabajo consiste en elaboración y presentación de un informe de buenas prácticas que recoja el modelo de intervención elaborado y que plantee mejoras en la práctica, vinculando de nuevo teoría y práctica.

La sistematización, por tanto, permite ordenar los aspectos calve del proceso y la reflexión realizada, esto queda plasmado en un documento que servirá de instrumento para la reflexión de todas las personas implicadas y que puede ser divulgado. También se realiza un" informe ejecutivo" en el que de manera sintética y esquemática se recogen los aspectos más significativos del trabajo realizado, así como las propuestas de mejora y buenas prácticas que han surgido del proceso de sistematización. Este documento permite sintetizar la experiencia y favorece su divulgación. Es a partir de aquí donde se establece la estrategia de comunicación, se divulga la experiencia y se desarrolla el plan de trabajo para la transferencia, mediante la divulgación en diferentes foros (Zaballa,2013).

\subsection{4 - A. Implementación de una Experiencia Piloto}

El modelo que estamos proponiendo abre también posibilidades para la puesta en marcha de una experiencia piloto con la finalidad de poner en marcha buenas prácticas, resultado de esa sistematización. Se trata de ofrecer un pilotaje, apoyo y acompañamiento por parte del equipo investigador para la puesta en práctica de la propuesta elaborada, en la que de nuevo participen activamente los agentes implicados en el proceso de manera que se abre un nuevo ciclo para sistematizar la experiencia realizada, para trabajar desde la facilitación, validar la práctica, e introducir ajustes y cambios necesarios que la mejoren. En definitiva, 
esta propuesta de sistematización contribuye a la construcción de conocimientos a partir de la práctica y de la realidad. Revisando los marcos de referencia, teóricos, metodológicos y conceptuales, de la propia intervención, enriqueciéndolos y replanteándolos.

\section{CONCLUSIONES}

Esta investigación constituye un aporte en dos esferas. En primer lugar, la aproximación desde la teoría y la metodología a lo más profundo de la práctica hace posible darse cuenta de la gran complejidad de la conjugación de ambas partes. Al tiempo que nos permite describir ese dinamismo argumental necesario para poder construir desde el conocimiento, un modelo de buenas prácticas, teniendo en cuenta los aspectos más importantes de ambas visiones, insostenibles la una sin la otra. En segundo lugar, disponer de un análisis real de la práctica para poder conducir procesos de mejora liderando esos cambios necesarios y propuestas de innovación donde los agentes implicados tomen parte y se impliquen activamente.

Tras este trabajo de Investigación Acción Participación (IAP), es decir, de escucha activa, de reflexión y estudio de las realidades y necesidades que se han ido describiendo y descubriendo; y siendo consecuentes con la Responsabilidad Social Universitaria (RSU), el equipo investigador propone liderar ese proceso de cambio, para que llegue a consolidarse el modelo o buena práctica resultante, ya que puede resultar una tarea compleja si no es conducida por expertos ajenos a la propia organización.

En definitiva, convertirse en facilitadores conduciendo los procesos de mejora desde una secuencia planificada (definición, identificación de fortalezas y áreas de mejora, priorización y temporalización de los cambios, puesta en marcha, visibilización, etc..). Acompañar desde el conocimiento teniendo en cuenta la información y la formación, y reflexionando sobre el propio trabajo.

\section{REFERENCIAS}

Amnistía Internacional (2011.) Manual de facilitación. Guía para el uso de metodologías participativas en la educación en Derechos Humanos.

Caparrós C., Carbonero D.\& Raya, E. (2017). Construir conocimiento desde la práctica: ejemplos de sistematización en Trabajo Social. Interacción y Perspectiva. Revista de Trabajo Social ISSN 2244-808X. 7 (1) pp. 61-79.

Costamagna P. \& M. Larrea (2017). Actores facilitadores del desarrollo territorial. Instituto Vasco de competitividad. Fundación Deusto. Donostia/San Sebastián 
Domínguez, M.J. (2009). Responsabilidad Social Universitaria. Humanismo y Trabajo Social Vol. (8) 37-67.

Ferran, A., Guinot, C., \& Berasategui, M.A., (2019). Gobernanza colaborativa en la inclusión social. Una experiencia de investigación y acción participativa. Investigación y Debate, 271. Ed. Catarata. Madrid.

Francke, M. \& Morgan M.L. (1995). La sistematización: apuesta por la generación de conocimientos a partir de las experiencias de promoción. Materiales Didácticos (1). Escuela para el desarrollo. Lima.

FAO/Mae (2004) Guía Metodológica de sistematización. En: tttp://www.fao.org/docs/eims/upload/190561/guiamet.pdf. Fecha de consulta: 08/02/20

Fucks S. (2009). FSPC: la facilitación sistémica de procesos colectivos. Artesanía de contextos focalizada en la promoción de la creatividad y de los procesos participativos en grupos, comunidades y redes. Revista IRICE, 20, 63-76.

Jara Holliday, O. (2012). Sistematización de experiencias, investigación y evaluación: Aproximaciones desde tres ángulos. Revista internacional sobre investigación en educación global y para el desarrollo, 1, 56-70.

Karlsen, J. \& Larrea, M. (2015). Desarrollo territorial e investigación acción. Publicaciones Deusto. Donostia.

León, O. \& Montero, I. (2003). Métodos de investigación en psicología y educación (3a . Edición). McGrawHill/Interamericana. Madrid.

Lewin, Kurt (1946). La investigación acción y los problemas de las minorías, 1, 15-26. La Investigación-acción participativa. Editorial Popular. Recuperado el 27/02/2020 desde:

http://www.eduneg.net/generaciondeteoria/files/Lewin\%20La\%20investigacionaccion $\% 20$ \%20los $\% 20$ problemas $\% 20$ de $\% 20$ las $\% 20$ minorias.pdf

Perlo, C., De la Riestra M.R. , Costa, L . (2009). Investigar el mal-estar, construir el saber estar para generar bien-estar en nuestros contextos organizativos. Revista IRICE, 20, 7-12.

Porter, M., \& Kramer, M.R (2011). La creación del valor compartido. Harvard Bussiness Review. America Latina. Enero- Febrero 2011. Extraído el 27.02.2020 desde: https://drive.google.com/file/d/1_141CJtVae5Gy3aUu7wtVKctlgdiOGTy/view

Sandín, M.P. (2003). Investigación cualitativa en Educación: Fundamentos y Tradiciones. Madrid: McGrawHill/Interamericana.

Segú, M., Gonzalez Goya, E. (2019) Transfrontier Exchange for Modelling Good Practices in Social Intervention Based on PAR: The Case of the Sarea Project. Fronteiras: Journal of Social, Technological and Environmental Science 8, (1). Extraído el 28.02.2020 desde:

Tapella,E. \& Rodriguez-Bilella ,P. (2014): Sistematización de experiencias: Una metodología para evaluar intervenciones de desarrollo. Revista de Evaluación de Programas y Políticas Públicas UNED, 3, 80-116.

Zaballa Caudillo,A. (2010). La sistematización desde una mirada interpretativa: propuesta metodológica. TRABAJO SOCIAL UNAM VI Época, 1, 90-101. Ciudad de México.

Zúñiga López, R.E. \& Zúñiga Preciado M.T. (2013). Metodología para la Sistematización Participativa de Experiencias Sociales. Una propuesta desde la Educación Popular IMDEC. Guadalajara. 